\title{
Distance between 2D-scenes based on Oriented Matroid Theory
}

\author{
Francesc Serratosa \\ Dept. d'Enginyeria Informàtica \\ Universitat Rovira i Virgili \\ Francesc.Serratosa@etse.urv.es
}

\author{
Antoni Grau \\ Automatic Control Dept \\ Technical Univ. of Catalonia, UPC \\ antoni.grau@upc.es
}

\author{
Alberto Sanfeliu \\ Inst. de Robòtica i Informàtica \\ Technical Univ. of Catalonia \\ asanfeliu@iri.upc.es
}

\begin{abstract}
In this paper a novel method for representing and comparing views of objects is presented. The topological properties of the regions of the views of objects are used to define a structure, called set of cocircuits, based on the oriented matroid theory. It is a formalism for qualitative spatial representation and reasoning and encodes information about relative position of the disjoint regions of the view and give local and global topological information about their spatial distribution. This topological technique is applied to recognising indoor scenes for the localization of a mobile robot.
\end{abstract}

\section{Introduction}

A fundamental problem in pattern recognition is to select suitable representations for objects and classes. In the decision-theoretic approach to pattern recognition, a pattern is represented by a set of numerical values, which forms a feature vector. Although, in many tasks, the recognition of objects can be achieved successfully using only global features such as size and compactness, in some applications, it is helpful to describe an object in terms of its basic parts and the relations between them. The most common techniques are based on the graph theory [7]. Nevertheless, another option is through the qualitative reasoning which it is based on comparative knowledge rather than on metric information. Many methods for shape representation and analysis are based on extracting points and edges which are used to define projectively invariant descriptors. In this paper, instead of points, regions of the images are taken into account. The motivation behind this choice is that the regions of an image can be more reliably extracted than edges.

Thus, the qualitative information of the topology of the image based on the regions is extracted, which is derived from the oriented matroid theory.

In the following sections a formalism for qualitative representation and comparing images is described. After that, we present some experimental results and the final conclusions.

\section{Oriented Matroids}

Oriented matroid theory [1], [2] and [3] is a broad setting in which the combinatorial properties of geometrical configurations can be described and analysed. It provides a common generalization of a large number of different mathematical objects usually treated at the level of usual coordinates. In this section oriented matroids will be introduced over arrangements of points using two combinatorial data structures called chirotope and set of cocircuits, which represent the main tools to translate geometric problems into this formalism. In the abstraction process from the concrete configuration of points to the oriented matroid, metric information is lost but the structural properties of the configuration of points are represented at a purely combinatorial level.

\subsection{Oriented Matroids of Arrangements of Points} Given a point configuration in $\Re^{\mathrm{d}-1}$ whose elements are the columns of the matrix $\mathbf{P}=\left(\mathrm{p}_{1}, \mathrm{p}_{2}, \ldots, \mathrm{p}_{\mathrm{n}}\right)$, the associated vector configuration is a finite spanning sequence of vectors $\left\{\mathrm{x}_{1}, \mathrm{x}_{2}, \ldots, \mathrm{x}_{\mathrm{n}}\right\}$ in $\mathfrak{R}^{\mathrm{d}}$ represented as columns of the matrix $\mathrm{X}=\left(\mathrm{x}_{1}, \mathrm{x}_{2}, \ldots, \mathrm{x}_{\mathrm{n}}\right)$ where each point $\mathrm{p}_{\mathrm{i}}$ is represented in homogeneous coordinates as $x_{i}=\left(\begin{array}{c}p_{i} \\ 1\end{array}\right)$.

To encode the combinatorial properties of the point configuration we can use a data structure called chirotope [3], which can be computed by means of the associated vector configuration $\mathrm{X}$. The chirotope of $\mathrm{X}$ is the map

$$
\begin{aligned}
\chi \mathrm{X}:\{1,2, \ldots, n\}^{d} & \rightarrow\{+, 0,-\} \\
\left(\lambda_{1}, \lambda_{2}, \ldots, \lambda_{d}\right) & \mapsto \operatorname{sign}\left(\left[x_{\lambda_{1}}, x_{\lambda_{2}},,,, x_{\lambda_{d}}\right)\right.
\end{aligned}
$$

that assigns to each d-tuple of vectors of the finite configuration $\mathrm{X}$ a sign + or - depending on whether it forms a basis of $\mathfrak{R}^{\mathrm{d}}$ having positive or negative orientation, respectively. This function assigns the value 0 to those d-tuples that do not constitute a basis of $\Re^{\mathrm{d}}$. The chirotope describes the incidence structure between the points of $\mathrm{X}$ and the hyper planes spanned by the same points and, at the same time, encodes the relative position of the points of the configuration with respect to the hyperplanes that they span.

Consider the point configuration $\mathrm{P}$ represented in figure 1 whose vector configuration $\mathrm{X}$ is given in Table 1 .

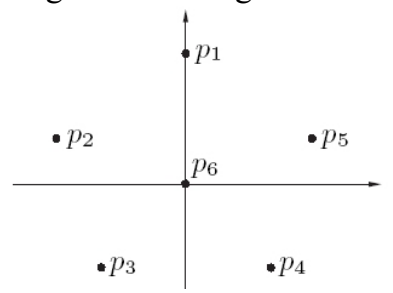

Fig. 1. A planar point configuration. 


\begin{tabular}{|c|c|c|}
\hline$x_{1}=(0,3,1)^{\mathrm{T}}$ & $x_{2}=(-3,1,1)^{\mathrm{T}}$ & $x_{3}=(-2,-2,1)^{\mathrm{T}}$ \\
\hline$x_{4}=(2,-2,1)^{\mathrm{T}}$ & $x_{5}=(3,1,1)^{\mathrm{T}}$ & $x_{6}=(0,0,1)^{\mathrm{T}}$ \\
\hline
\end{tabular}

Table 1. Vector configuration that corresponds to the planar point configuration represented in figure 1.

The chirotope $\chi \mathrm{X}$ of this vector configuration is given by the orientations listed in Table 2 . The element $\chi(1,2,3)=$ + indicates that in the triangle formed by $\mathrm{p}_{1}, \mathrm{p}_{2}$ and $\mathrm{p}_{3}$ these points are counter clockwise ordered. These orientations can be rearranged in an equivalent data structure called set of cocircuits of X shown in Table 3. In this planar case, the set of cocircuits of $X$ is the set of all partitions generated by the lines passing through two points of the configuration.

\begin{tabular}{l|l|l|l|l|}
$\chi(1,2,3)=+$ & $\chi(1,2,4)=+$ & $\chi(1,2,5)=+$ & $\chi(1,2,6)=+$ & $\chi(1,3,4)=+$ \\
\hline
\end{tabular} \begin{tabular}{|l|l|l|l|l|}
\hline$\chi(1,3,5)=+$ & $\chi(1,3,6)=+$ & $\chi(1,4,5)=+$ & $\chi(1,4,6)=-$ & $\chi(1,5,6)=-$ \\
\hline$\chi$
\end{tabular} \begin{tabular}{|l|l|l|l|l|}
\hline$\chi(2,3,4)=+$ & $\chi(2,3,5)=+$ & $\chi(2,3,6)=+$ & $\chi(2,4,5)=+$ & $\chi(2,4,6)=+$ \\
\hline
\end{tabular} \begin{tabular}{|l|l|l|l|l|}
\hline$\chi(2,5,6)=-$ & $\chi(3,4,5)=+$ & $\chi(3,4,6)=+$ & $\chi(3,5,6)=+$ & $\chi(4,5,6)=+$ \\
\hline
\end{tabular}

Table 2. Chirotope of the planar point configuration represented in figure 1 .

\begin{tabular}{|c|c|c|}
\hline$(0,0,+,+,+,+)$ & $(0,-, 0,+,+,+)$ & $(0,-,-, 0,+,-)$ \\
\hline$(0,-,-,-, 0,-)$ & $(0,-,-,+,+, 0)$ & $(+, 0,0,+,+,+)$ \\
\hline$(+, 0,-, 0,+,+)$ & $(+, 0,-,-, 0,-)$ & $(+, 0,-,-,+, 0)$ \\
\hline$(+,+, 0,0,+,+)$ & $(+,+, 0,-, 0,+)$ & $(+,+, 0,-,-, 0)$ \\
\hline$(+,+,+, 0,0,+)$ & $(-,+,+, 0,-, 0)$ & $(-,-,+,+, 0,0)$ \\
\hline
\end{tabular}

Table 3. Set of cocircuits of the planar point configuration represented in figure 1.

For example, $(0,0,+,+,+,+)$ means that the points $\mathrm{p}_{3}, \mathrm{p}_{4}$, $\mathrm{p}_{5}$, and $\mathrm{p}_{6}$ lie on the half plane determined by the line through the points $\mathrm{p}_{1}$ and $\mathrm{p}_{2}$. Reversing all the signs of the set of cocircuits we obtain an equivalent description of the planar arrangement of points.

Besides chirotopes and cocircuits there are several data structures capable of encoding the topological properties of a point configuration. In [3] their definitions can be found and it is shown that all of them are equivalent and are referred to as oriented matroids.

\subsection{Oriented Matroid of Arrangement of Regions} Consider a segmented view of a 3D object. Extracting the oriented matroid of a view is not straightforward since the regions that form the image cannot be reduced to points, taking for instance their centroids, without losing essential topological information for object recognition. Therefore, the convex hull [4] of each region is employed to represent the region itself. Then, pairs of the resulting convex polygons are considered and the oriented matroid is computed based on the spatial location of the other convex regions of the image with respect to the two lines arising in merging the convex hulls of pairs disconnected regions.

Consider, for instance, the ordered pair of convex regions $(S, T)$ of figure 2.a. It is easy to see that the convex hull of these two planar convex disconnected polygonal regions is a polygon whose set of vertices is included in the union of the set of vertices of $S$ and $T$. On the contrary, the set of edges of the convex hull of $S$ and $T$ is not included in the union of their set of edges. Indeed, two new "bridging edges," $e_{1}$ and $e_{2}$, appear as illustrated in figure 2.a. Actually, efficient algorithms for merging convex hulls are based on finding these two edges [5].

Consider the two lines $l_{1}$ and $l_{2}$ that support $e_{1}$ and $e_{2}$. These two lines divide the image into three or four zones depending on the location of their intersection point with respect to the image. Let $R_{S, T}$ and $L_{S, T}$ (figure 2.b) be, respectively, the rightmost and leftmost zones with respect to $l_{1}$ and $l_{2}$ and $I_{S, T}$ the zone of the image comprised between them.

Since $R_{S, T}, L_{S, T}$ and $I_{S, T}$ can be univocally determined from the ordered couple of region $(S, T)$, the location of a region $U$ with respect to the regions $(S, T)$ of the image is encoded into a chirotope using the following rule

$$
\chi(S, T, U)=\left\{\begin{array}{l}
+ \text { if } U \in L_{S, T} \\
0 \text { if } U \in I_{S, T} \\
- \text { if } U \in R_{S, T}
\end{array}\right.
$$

It has been implicitly assumed that $U$ is completely contained into either $R_{S, T}, L_{S, T}$ or $I_{S, T}$ but, in general, it belongs to more that one of them. In this case, since the ratio of areas is an affine invariant, introducing an approximation, we can choose the sign based on which region contains the largest portion of the area of $U$. For instance, if regions $U, V$ and $Z$ are located as in figure 2.c we have that $\chi(S, T, U)=+, \chi(S, T, V)=0$ and $\chi(S, T, Z)=-$.

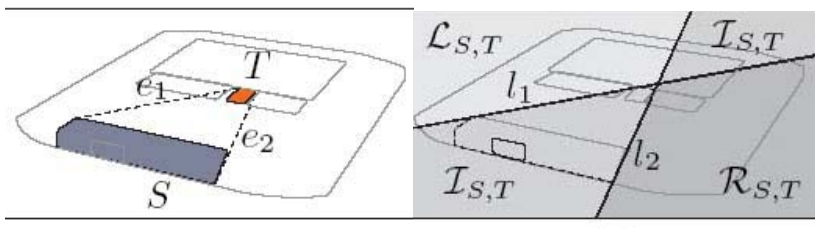

(a)

(b)

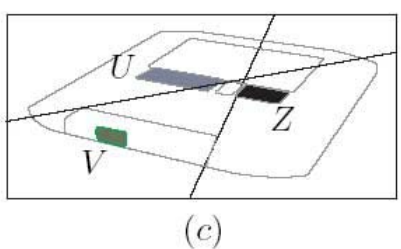

Fig. 2. Steps of encoding of the combinatorial properties of a view of an object into a chirotope, (a) the bridging edges $\left(e_{1}\right.$ and $e_{2}$ ) are extracted from the convex hulls of the regions $S$ and $T$, (b) the zones $R_{S, T}, L_{S, T}$ and $I_{S, T}$ are considered and finally, (c) the topological situations of the other regions ( $U, V$ and $Z$ ) are obtained. 


\section{Distance measure between sets of cocircuits} Let $T^{1}=\left\{C_{1}^{1}, \ldots C_{r^{1}}^{1}\right\}$ and $T^{2}=\left\{C_{1}^{2}, \ldots C_{r^{2}}^{2}\right\}$ be two sets of cocircuits representing images that contain $r^{1}$ and $r^{2}$ cocircuits, respectively and the same combinations $s$ of regions. Moreover, let the cocircuits defined as a vector of signs $C_{i}^{k}=\left(c_{i, 1}^{k}, \ldots c_{i, s}^{k}\right)$ in which, each sign is defined as $c_{i, j}^{k}=\{\nexists,+, 0,-\}$ where $k=\{1,2\}$.

A distance measure is presented in this section to provide a quantitative value of the match between two sets of cocircuits $T^{l}$ and $T^{2}$. To this aim, we first define the null cocircuit $\mathrm{C}_{\Phi}$ and the distance between cocircuits $d c$.

\subsection{The null cocircuit}

The null cocircuit is used to represent the non-existence of a cocircuit in one set of cocircuits. It is defined as $C_{\Phi}=(\Phi, \ldots \Phi)$ and it has $s$ null elements, $\Phi$, that is, the number of combinations of regions that the set of cocircuits has.

\subsection{Distance between cocircuits}

The distance measure between a pair of cocircuits $C_{i}^{1}$ and $C_{j}^{2}$ with $s$ combinations of regions each one is defined as the addition of a cost applied on all the pair of elements of the cocircuit.

$$
\operatorname{dist}\left(C_{i}^{1}, C_{j}^{2}\right)=\sum_{g=1}^{s} \operatorname{Cost}\left(C_{i, g}^{1}, C_{j, g}^{2}\right)
$$

where the function Cost represents the spatial situation between the regions.

For instance, the following tables could be a choice:

\begin{tabular}{|c|c|c|c|c|c|}
\hline Cost & + & - & 0 & $\nexists$ & $\Phi$ \\
\hline+ & 0 & 2 & 1 & 3 & 4 \\
\hline- & 2 & 0 & 1 & 3 & 4 \\
\hline 0 & 1 & 1 & 0 & 3 & 4 \\
\hline$\nexists$ & 3 & 3 & 3 & 0 & 4 \\
\hline$\Phi$ & 4 & 4 & 4 & 4 & 0 \\
\hline
\end{tabular}

\subsection{Distance between sets of cocircuits}

The distance between cocircuits is somehow related to the topological similarity of a labelling function $f: T^{l} \rightarrow T^{2}$ given the set of cocircuits $T^{l}$ and $T^{2}$ extracted from a pair of segmented images. We may attempt to minimise a global cost measure $k_{f}$ of a morphism $f$ in the set $H$. This set represent the allowable configurations or labellings.

Once a cost measure $k_{f}$ is defined, a distance measure and the optimal labelling $f^{*}$ are defined respectively as

$$
d=\min _{f \in H}\left\{k_{f}\right\} \text { and } f^{*}=\arg \min _{f \in H}\left\{k_{f}\right\}
$$

Moreover, for the sake of robustness, the mapping $f$ will not be defined from the initial $T^{l}$ and $T^{2}$, but from the $k$ extended $T^{l}$ to the $k$-extended $T^{2}$, to contemplate the possibility of some missing cocircuits or some extraneous cocircuits introduced by noisy effects. A missing cocircuit in $T^{l}$ will be represented by a null cocircuit $C_{\Phi}=(\Phi, \Phi, \ldots \Phi)$ in the extended $T^{l}$, and an extraneous cocircuit in $T^{l}$ should be mapped to a null cocircuit in the extended $T^{2}$. The number of cocircuits $k$ in the extended cocircuits is theoretically set to the sum of the number of cocircuits in both sets of cocircuits. Hence, the limit situations in which all the cocircuits in $T^{2}$ are missing in the $T^{l}$ or all the cocircuits in the $T^{l}$ are extraneous are covered.

Let $T^{, l}$ and $T^{, 2}$ be the k-extension of the cocircuits $T^{l}$ and $T^{2}$ with the same number of cocircuits $k$ and the domain of the signs including the null value, $c_{i, j}^{k}=\{\nexists,+, 0,-, \Phi\}$.

Thus, the set $H$ of allowable labelling functions $f: T^{, l} \rightarrow T^{, 2}$ is actually defined as a the subset of bijective functions from $T^{, l}$ to $T^{, 2}$.

The global cost $k_{f}$ of a morphism $f$ is defined as

$$
k_{f}=\sum_{i=1}^{s} d c\left(C_{i}^{1}, C_{f(i)}^{2}\right)
$$

where $d c$ is the distance between a pair of cocircuits belonging to the original set of cocircuits $\left(T^{, l}\right.$ or $\left.T^{, 2}\right)$ or the extended ones.

\section{Oriented Matroids to represent 3D-objects}

The process of representing 3D-objects by the Oriented Matroid theory starts with some preliminary choices, namely the features used to characterize the regions of the segmented views of the set of 3D objects. Suppose that hue and area are used to characterize each region. Another parameter to choose is the number of levels in which the hue is quantized and the number of regions having the same hue that will be taken into account. These choices, of course, depend on the properties of the views of the database. Then, the views are segmented according to these choices and the convex hull of each region is computed. As a consequence, the resulting images are compositions of convex polygonal regions that can be disconnected or partially or completely overlapped. In figure 3 are represented two views of two objects in which a hue quantization with 6 levels $\mathrm{W}, \mathrm{R}, \mathrm{Y}, \mathrm{G}, \mathrm{B}$ and $\mathrm{N}$ has been applied and only the two biggest regions with the same hue value are taken into account.

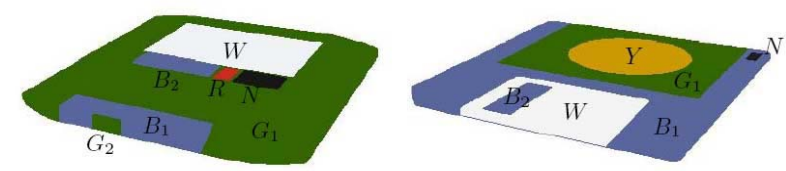

Fig. 3. Two objects whose cocircuits are shown in Table 4.

Let $(\mathrm{W}, \mathrm{R}, \mathrm{Y}, \mathrm{G}, \mathrm{B}, \mathrm{N})$ be the ordered tuple of hue levels considered. For example, labels G1 and G2 in Figure 3 denote, respectively, the first and the second regions of the views with the biggest area having the hue value $\mathrm{G}$. 


\begin{tabular}{|c|c|c|c|c|c|c|c|c|c|c|}
\hline Cocirc. & & $W$ & $R$ & $Y$ & $G_{1}$ & $G_{2}$ & $B_{1}$ & $B_{2}$ & $N$ & Obj. \\
\hline$C_{1}^{1}$ & $W R$ & 0 & 0 & $\nexists$ & 0 & 0 & 0 & - & + & 1 \\
\hline$C_{2}^{1}$ & $W G_{2}$ & 0 & 0 & $\nexists$ & 0 & 0 & + & 0 & 0 & 1 \\
\hline$C_{3}^{1}$ & $W B_{1}$ & 0 & 0 & $\nexists$ & 0 & 0 & 0 & 0 & 0 & 1 \\
\hline$C_{4}^{1}$ & $W B_{2}$ & 0 & 0 & $\nexists$ & + & + & + & 0 & + & 1 \\
\hline$C_{5}^{1}$ & $W N$ & 0 & 0 & $\nexists$ & - & - & - & - & 0 & 1 \\
\hline$C_{1}^{2}$ & $W Y$ & 0 & $\nexists$ & 0 & 0 & $\nexists$ & 0 & 0 & - & 2 \\
\hline$C_{2}^{2}$ & $W G_{1}$ & 0 & $\nexists$ & 0 & 0 & $\nexists$ & 0 & 0 & 0 & 2 \\
\hline$C_{3}^{2}$ & $W N$ & 0 & $\nexists$ & + & + & $\nexists$ & 0 & 0 & 0 & 2 \\
\hline
\end{tabular}

Table 4. Set of cocircuits of the objects in figure 3 .

When $S$ and $T$ are disconnected, the corresponding cocircuit is present in the index. The symbol " $\nexists$ " in correspondence with a certain feature indicates that no region with that feature is present in the views listed in the record. For example, the cocircuit WR contains a $\nexists$ in the column $\mathrm{Y}$ because no region with the $\mathrm{Y}$ feature is present in the object. If $(S, T)$ is a couple of connected regions or $\mathrm{S}$ or $\mathrm{T}$ does not exist, the cocircuit cannot be computed and so it does not appear in the set of cocircuits.

Table 5 shows the distance values between all the cocircuits of objects in figure 3. The bold numbers represent the labelling $f^{*}$ that gives the minimum cost.

\begin{tabular}{|c|c|c|c|c|c|}
\hline $\operatorname{dist}\left(C_{i}^{1}, C_{j}^{2}\right)$ & $C_{1}^{1}$ & $C_{2}^{1}$ & $C_{3}^{1}$ & $C_{4}^{1}$ & $C_{5}^{1}$ \\
\hline$C_{1}^{2}$ & 12 & $\mathbf{1 1}$ & 10 & 13 & 13 \\
\hline$C_{2}^{2}$ & 11 & 10 & $\mathbf{9}$ & 12 & 12 \\
\hline$C_{3}^{2}$ & 12 & 11 & 10 & $\mathbf{1 1}$ & 13 \\
\hline
\end{tabular}

Table 5. Distance values between the cocircuits of both objects.

\section{Application}

The Oriented Matroid theory is applied on positioning a mobile robot on indoor places by recognising the objects that are near it using computer vision.

Figure 4 shows a selection of 8 images used to built the index of cocircuits table. The $74 \%$ of the images where correctly recognised. Some images were wrongly recognised (recognised as it was another image) and another ones where not recognised (no image was considered similar enough). These errors were basically due to the change of the hue of the regions and the change of the point of view. Nevertheless, when the vision system cannot recognise the image (not recognised) or considers that the image that it views is far from it is supposed to be (wrongly recognised), the control of the robot supposes that its position is the same than the last reliable one.
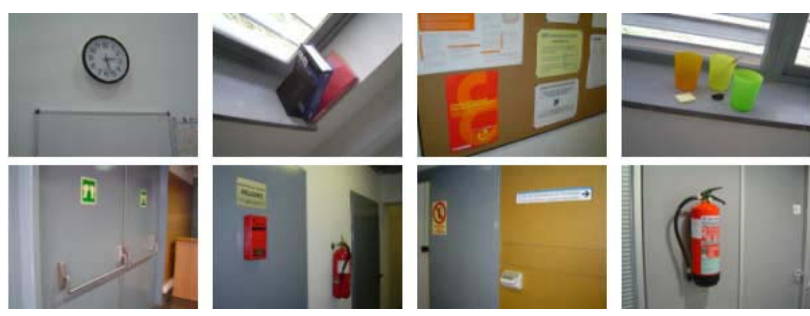

Fig. 4. Some images taken from the mobile robot Marco.

\section{Conclusions}

In this paper a new method for representing views of 3D object has been presented. It is based on a qualitative representation derived from the oriented matroid theory. This qualitative representation characterizes the local and global topology of the regions of an image, is invariant under affine and Euclidean transformation of the views, intrinsically robust to discretization errors of the image and insensitive to small displacements of the point of view.

We have applied the method on recognising indoor scenes of 3D-objects and the experimental results have shown the usefulness of the method due to the high ratio of correctness and also the run time.

\section{References}

[1] Bjorner, A., Vergnas, M.L., Sturmfels, B., White, N., Ziegler, G.M.: "Oriented Matroids". Volume 43 of Encyclopedia of Mathematics and its Applications. Cambridge University Press (1993).

[2] Bokowski, J., Sturmfels, B.: "Computational Synthetic Geometry". Volume 1355 of Lecture Notes in Mathematics. Springer-Verlag (1989).

[3] Richter-Gebert, J., Ziegler, G.M.: "Oriented matroids". Goodman, J.E., O'Rourke, J., eds.: Handbook of Discrete and Computational Geometry. CRC Press (1997)

[4] O'Rourke, J.: "Computational Geometry in C". Cambridge University Press (1999).

[5] Toussaint, G.T.: "Solving geometric problems with the rotating calipers". Proceedings of IEEE MELECON'83, Athens, Greece (1983).

[6] Staffetti, E., Grau, A., Serratosa, F. and Sanfeliu, A., "Shape Representation and Indexing based on Region Connection Calculus and Oriented Matroids Theory", 11th International Conference on Discrete Geometry for Computer Imagery, DGCI 2003, LNCS 2886, pp.267-276.

[7] Serratosa, F., Alquézar, R., and Sanfeliu, A. "Function-described for modelling objects represented by attributed graphs", Pattern Recognition, 36 (3), pp. 781798, 2003. 